\title{
ANÁLISIS DE LA APLICACIÓN DA LA NORMATIVA BRASILEÑA NBR 15575 Y ALGUNAS ESTRATEGIAS PARA AUMENTAR LA CALIDAD AMBIENTAL EN EDIFICIOS HABITACIONALES
}

\section{ANALYSIS OF APPLICATION OF THE BRAZILIAN STANDARD NBR 15575 AND SOMESTRATEGIES TO INCREASE ENVIRONMENTAL QUALITY IN RESIDENTIAL BUILDINGS}

\author{
BRUNA LILIANE BRENNER, Ms.C. | UNISINOS \\ MATHEUS SBARDELOTTO BALDO | UNISINOS \\ MARCO AURELIO STUMPF GONZALEZ, Dr. | UNISINOS \\ ANDREA PARISI KERN, Dra.| UNISINOS
}

\begin{abstract}
RESUMEN
Algunos países tienen directrices para ampliar el desempeño en los edificios con el fin de proporcionar más comodidad a los usuarios y aumentar la calidad y eficiencia de los edificios. En Brasil, la evaluación del desempeño de los edificios se consolidó con la NBR 15575. Esta normativa establece diferentes criterios para el diseño de un edificio residencial. Sus requisitos han sido estudiados en el mundo académico, pero aún se requiere una evaluación de cómo los profesionales del sector de la construcción han recibido estos nuevos requisitos, el proceso de adaptación y cómo han aplicado en sus proyectos. Para llevar a cabo esa evaluación se envió un cuestionario a ingenieros civiles y arquitectos con actividad profesional en la región de Porto Alegre, Brasil. Se hice el análisis de la popularidad de las estrategias para ampliar el desempeño térmico, acústico y lumínico propuestas por la normativa. Los resultados mostraron que, aun que los profesionales tengan retos que superar, el desempeño ambiental se ha hecho más popular no sólo entre los profesionales, sino que también entre los clientes. Este factor es de gran importancia para el desarrollo y consolidación de la visada sobre desempeño en el mercado de la construcción.
\end{abstract}

PALABRAS CLAVE: Construcción civil; edificaciones, desempeño, NBR15575, profesionales.

\begin{abstract}
Some countries have guidelines for expanding performance in order to provide more comfort to users and increase the quality and efficiency of buildings. In Brazil, the evaluation of building performance was consolidated with the NBR 15575. This regulation establishes some criteria to develop the design of residential buildings. Its requirements have been studied in the academic world, but still it is important an evaluation of how construction professionals have received these new requirements, their adaptation to the normative and how they have applied in their projects. To carry out this evaluation we applied a questionnaire to civil engineers and architects with professional activity in the region of Porto Alegre, Brazil. We develop an analysis about the popularity of strategies to increase thermal, acoustic and luminous performance as proposed by this normative. The results shown that, although professionals still have some challenges, the environmental performance has become more popular not only among professionals, but also among clients. It is very important for the development and consolidation of performance view in the construction market.
\end{abstract}

KEY WORDS: Civil construction; buildings; performance; NBR15575, professionals. 


\section{INTRODUCCIÓN}

El concepto de desempeño es conocido en el sector de la construcción hace algún tiempo. La definición más comúnmente aceptada afirma que el desempeño es la práctica de pensar en términos de fines y no de medios. Es decir, pensar en los requisitos que debe cumplir el edificio (BORGES, 2008). La normativa brasileña NBR 15575 sigue este concepto. No es una norma prescriptiva, y por lo tanto no indica cómo el edificio debe ser construido, pero el desempeño que se debe alcanzar, independientemente de su sistema de construcción (ABNT, 2013a).

Para Gonçalves et al. (2007), el desempeño puede ser definido como el comportamiento en el uso de un producto. Así, debe tener propiedades que realizan la función indicada cuando se someten a ciertas influencias o acciones (condiciones de exposición) a lo largo de su vida útil. Por lo tanto, analizar el desempeño de un producto requiere especificar cualitativa y / o cuantitativa cuales son los requisitos previos que el producto debe alcanzar cuando se somete a un uso normal y los medios para evaluar si se cumplen las condiciones establecidas.

La NBR 15575 tiene por objeto establecer normas mínimas de calidad requeridos para algunos sistemas de construcción, tales como estructuras, pisos interiores, paredes y cercas, techos y sistemas sanitarios durante la vida útil de un edificio. Su ámbito de aplicación es amplio, y hay el establecimiento de requisitos mínimos de desempeño para los sistemas de los edificios, consideradas las necesidades del usuario y las condiciones de exposición edificio (ABNT, 2013a; LAMBERTS et al., 2010). La NBR 15575 hice algunos cambios en el sector de construcción en Brasil, como la aplicación de una nueva metodología de diseño del edificio. La necesidad de adaptación hice cambios en varios sectores relacionados con el proyecto y desarrollo del edificio, tales como diseñadores, industria, proveedores y constructores (KERN et al, 2015).

Bento et al. (2016) investigaron la comprensión y el interés de los ingenieros en la norma de desempeño, con una mirada al impacto potencial sobre la durabilidad de los edificios. Los resultados muestran que la mayoría de los profesionales comprende que la aplicación de la NBR15575 puede beneficiar a la industria, reducir los problemas futuros y extender la vida útil de los edificios.

Flach et al. (2017) investigaron el interés de los profesionales en el desempeño ambiental, centrándose en los aspectos acústicos y térmicos. El análisis de las experiencias de arquitectos e ingenieros involucrados en la investigación se llevó a cabo a través de un cuestionario. Los resultados indican que existe aún la necesidad de una mayor apreciación de los aspectos de desempeño del proyecto.
La normativa de desempeño también aumentó la popularidad de los conceptos de desempeño en los edificios dentro y fuera de la academia. Como se ha visto, algunos estudios se han realizado en torno a los requisitos de la norma, pero aún hay dudas acerca de la inclusión de contenidos estudiados en las escuelas en los proyectos de construcción reales debido a las dificultades encontradas por el sector para adaptarse a las nuevas exigencias.

Este artículo tiene como objetivo analizar la popularidad de las prácticas de diseño destinadas a mejorar los desempeños térmico, acústico y lumínico en la rutina profesional de arquitectos e ingenieros civiles que actúan en la región de Porto Alegre, una ciudad en el sur de Brasil. Para este fin se enviaron cuestionarios a los profesionales en diferentes ciudades de esta región preguntando sobre su conocimiento de la norma, conceptos y requisitos necesarios para obtenerse el desempeño ambiental y la aplicación en proyectos llevados a cabo por ellos.

\section{DESEMPEÑO AMBIENTAL}

Para el Instituto de Investigación Tecnológica (IPT), el desempeño ambiental es la conformidad de los ambientes internos a un conjunto de requisitos establecidos en función de las necesidades humanas, respetando las condiciones térmicas, de luminosidad, de ventilación, el aislamiento acústico, y la calidad visual y táctil (IPT, 2016).

El desempeño ambiental en edificios residenciales a lo largo de su período de funcionamiento es uno de los requisitos establecidos por la NBR 15575 a través de los requisitos relacionados con el desempeño térmico, acústico y lumínico (ABNT, 2013a).

Para los arquitectos e ingenieros, el desempeño ambiental de los edificios debe tener en cuenta también la planificación urbana y la construcción de edificios con sus instalaciones. En cada uno de ellos hay dos etapas diferentes: diseño y ejecución. En la fase de diseño de las soluciones son más fáciles y económicas. El desarrollo de un buen diseño y el uso de componentes con mejor desempeño es esencial para proporcionar al final un buen desempeño para los edificios (IPT, 2016).

El concepto de desempeño ambiental implica carácter fisiológico variable humana, psicológica y económica que deben cumplir por el edificio para que cumpla la función para la que fue diseñado durante su vida útil (MITIDIERI FILHO y HĖLENE, 1998). Las variables relacionadas con la seguridad, la comodidad, la funcionalidad y otros aspectos son analizadas y posteriormente generan criterios de desempeño que se deben considerar en el diseño e implementación de un edificio (HOPFE, 2009 apud KERN et al., 2015). 
Basado en la normativa, la aplicación de conceptos de desempeño ambiental en el proyecto consiste en la mejora de aspectos como el confort térmico, acústico y lumínico (CORBELLA y YANNAS, 2009). A pesar de este hecho, en general la actividad de proyecto por parte de los desarrolladores de bienes raíces está ordenada principalmente para reducir el coste de diseño y ejecución de la obra. El fin principal del desarrollador es el lucro y a menudo deja al fondo en el desempeño ambiental y otros elementos relacionados con la calidad de la edificación (OKAMOTO, 2015).

El desempeño ambiental en los espacios internos es de gran importancia para los usuarios ya que puede tener gran influencia sobre la calidad de vida. El malestar al usuario se puede producir por el frío, el calor, ruidos, poca o demasiado luminosidad y por lo tanto efectos negativos pueden aparecer como la interferencia con el desempeño de las tareas, dolores de cabeza de tensión, entre otros (FERRAZ, 2008).

La aplicación de estrategias para ampliar el desempeño ambiental de los edificios depende de factores tales como el conocimiento y la formación de profesionales. Las estrategias para la expansión del desempeño ambiental se aplican más fácilmente en la etapa de diseño. Las elecciones adecuadas en esta etapa son cruciales para el desempeño de la construcción (IPT, 2016).

\subsection{Desempeño acústico}

El problema del ruido en las zonas urbanas se ha incrementado debido al crecimiento de las ciudades y el aumento en el número de vehículos e industrias. De acuerdo con la Organización Mundial de la Salud (OMS), después de la contaminación atmosférica procedente de las emisiones de gases y la contaminación del agua, la contaminación acústica es lo que más afecta las personas en todo el mundo (OMS, 2003).

La NBR 15575 determina criterios de exposición a el ruido audible, clasificando las edificaciones en niveles superior, medio y mínimo. Eso ocurre a través del análisis de aislamiento de ruido aéreo en las paredes y ventanas, y del ruido de percusión a través de la prueba de percusión en las losas. La normativa también propone las formas de verificación (por ensayos de campo o laboratorio) y control. Edificios bien construidos deben ofrecer un desempeño acústico en todos los ambientes internos (ABNT, 2013a).

La calidad de sonido de los espacios internos es muy importante, ya que puede influir en la calidad de vida, el sueño y las relaciones entre usuarios de un mismo edificio. Para Ferraz (2008), cuando la calidad de un medio se deteriora, los efectos negativos pueden aparecer, tales como la interferencia con el desempeño de las tareas y los problemas de salud: irritabilidad, hipertensión, dolores de cabeza, insomnio, estrés. La exposición al ruido durante largos períodos, como el ruido de las tuberías y de impacto en los pisos, puede causar efectos secundarios perjudiciales para la salud.

Según Lopes (2010), hay varias estrategias de proyecto para controlar el ruido, las cuales incluyen desde la eliminación de la fuente de ruido por la elección de la posición de edificio en relación con el entorno hasta el material de cierre, el tamaño y la colocación de las aberturas. Las paredes y los techos, particiones y ciertos materiales de construcción tienen la capacidad de atenuar la transmisión del sonido de un ambiente a otro. La condición acústica de los ambientes puede ser influenciada por los detalles de construcción, el acabado superficial y pequeños cambios en el diseño. Cada elección realizada en cuanto a estructuras, cubiertas, materiales de revestimiento, sistemas de climatización y otros, cambia la forma del edificio el comportamiento del sonido en el interior del edificio.

\subsection{Desempeño térmico}

La normativa brasileña NBR 15575 describe el rendimiento térmico de un edificio como un conjunto de características que satisfacen los requisitos de confort térmico de los usuarios (ABNT, 2013a). Para Lamberts y Triana (2007), rendimiento térmico es la capacidad de proporcionar confort térmico al usuario de forma pasiva y se determina por las características de la envolvente, como los colores y materiales empleados, la orientación solar, el uso o no de aislamiento o estrategias bioclimáticas. Un buen rendimiento térmico puede representar una reducción significativa del consumo de energía en un edificio, y se reduce la necesidad de utilizar sistemas de artificiales de aire acondicionado.

Según Frota y Schiffer (2001), para que se permitan condiciones de confort térmico a los usuarios, el edificio debe ser apropiado para el clima en el que opera, suavizando las sensaciones impuestas por el clima local. Para Cunha et al. (2006), el clima es uno de los elementos fundamentales para el consumo de energía necesario para mantener el edificio en buenas condiciones de desempeño térmico.

Los criterios mínimos de desempeño térmico están estandarizados en Brasil por las normativas NBR 15220 y NBR 15575. A nivel internacional, está estandarizado por las normas ISO 7730, ANSI/ASHRAE 50, ISO 7730, ISO 11079, ACGH y OSHA, entre otras (ABNT, 2013a; ABNT, 2013b; LAMBERTS et al., 2007). Para la NBR 15575, se puede clasificar cualitativamente el desempeño térmico, con los criterios de evaluación de los valores máximos en verano 
y la temperatura interna mínima en invierno. La norma establece tres procedimientos de evaluación: prescriptivo, por simulación por ordenador y medición de la construcción o prototipo (ABNT, 2013a).

\subsection{Desempeño lumínico}

La iluminación artificial permite la realización de actividades a la noche o en ambientes cerrados, pero nunca debe sustituir totalmente la luz natural, pues esa proporciona beneficios psicológicos, fisiológicos, ambientales y económicos. La iluminación natural se encuentra disponible en la mayoría de las horas del día, pero es necesario que el diseñador tenga conocimiento de las condiciones locales para su correcto uso en el edificio (LAMBERTS et al., 2014). En el caso de la utilización de estrategias de iluminación natural algunas precauciones son necesarias, ya que ella también puede influir en el rendimiento térmico del edificio (MACEDO, 2002).

El confort visual del usuario es un factor importante que considerar en la definición de las necesidades de iluminación en el interior del edificio. La iluminación debe tener dirección e intensidad adecuadas para el lugar, así como proporcionar una buena definición visual y ausencia de reflejos (LAMBERTS et al., 2014). En Brasil, la NBR ISO/

CIE 8995-1 establece los valores mínimos de luminancia en servicio para iluminación artificial en el interior de los edificios donde se llevan a cabo actividades de comercio, industria y educación (ABNT, 2013c). Para la iluminación natural en el interior de los edificios los criterios mínimos se establecen por NBR 15215 (ABNT, 2013b). Por fin, los valores mínimos de iluminación artificial y natural dentro de los ambientes domésticos son establecidos por NBR 15575 (ABNT, 2013a).

\section{MÉTODO EMPLEADO}

En el análisis del conocimiento y de la aplicación de los conceptos relacionados con el desempeño ambiental por parte de los profesionales se llevó a cabo desde el envío de cuestionarios los arquitectos e ingenieros civiles que trabajan en la región de Porto Alegre, Brasil.

El cuestionario tuvo como objetivo analizar la popularidad de aplicación de estrategias de desempeño térmico, acústico y lumínico en esta región. Se les solicitó informaciones básicas del profesional, tales como edad, tiempo de grado y tipo de formación universitaria, formación complementaria y área de práctica profesional.

A continuación, se propusieron cuestiones relacionadas con el conocimiento y la aplicación de la norma NBR 15575 y las estrategias empleadas para la expansión del desempeño térmico, acústico y lumínico. Por fin, se investigó la importancia dada por los clientes y por los propios profesionales sobre estos aspectos.

El cuestionario fue respondido por 70 profesionales, con 35 arquitectos y 35 ingenieros civiles que trabajan en el mercado de la construcción en las actividades de proyecto y ejecución de obras. Algunos de éstos, también tienen actividad de la enseñanza o la investigación.

\section{PRESENTACIÓN Y DISCUSIÓN DE RESULTADOS}

Todos los profesionales actúan en diseño y/o ejecución. Una serie de 45 encuestados (80,4\%) actúa en diseño, y 26 $(46,4 \%)$ en ejecución de obras. Un $9 \%$ de los mismos también lleva a cabo la actividad docente; 12,5\% tiene actividad de investigación y $12,5 \%$ desarrolla otras actividades profesionales. Además del área de actuación en el mercado, los encuestados respondieron sobre su formación complementaria. De ellos, 4,3\% poseen doctorado, 21,4\% tienen maestría, 27,1 tienen un título de especialización, y $47,1 \%$ no poseen formación complementaria.

A partir de las preguntas relacionadas con el comportamiento ambiental, los encuestados dijeron cuales actividades habían sido llevadas a cabo en los proyectos que tenían trabajado. Los resultados se muestran en la Figura 01. La mayoría de los profesionales (72,8\%) afirma que si, han participado en los proyectos que se implementaron estrategias para ampliar el desempeño térmico. Todavía un 17,1\% de los encuestados nunca han participado de proyectos con esta preocupación.

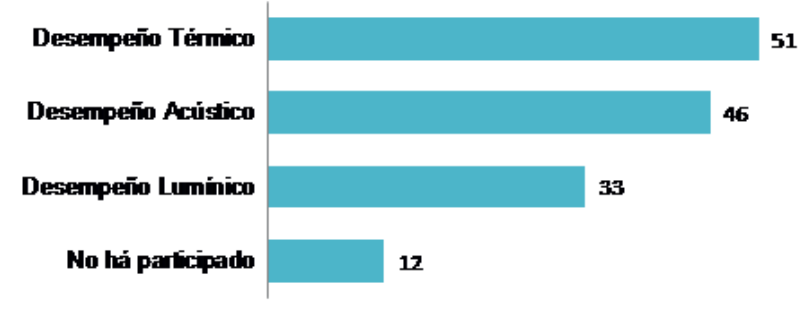

Figura 01 - Tipos de análisis de desempeño realizados por los profesionales Fuente: Autores.

Para los profesionales que participaron en proyectos en que se han aplicado estrategias para expansión de desempeño ambiental, se solicitó la especificación do que se había utilizado. Las respuestas a esta pregunta se resumen en la Figura 02 y se puede observar que la estrategia más popular es el uso de aislantes térmicos, seguido por el análisis de la posición solar del edificio. 


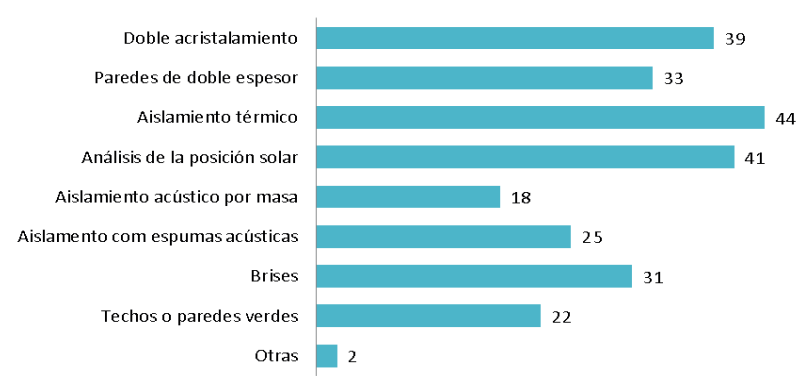

Figura 02 - Especificación de las estrategias utilizadas por los profesionales Fuente: Autores.

Una herramienta importante para analizar el desempeño ambiental de los edificios son los softwares de simulación. No obstante, algunos investigadores, como Palos et al. (2014) y Barison y Santos (2016) afirman que aún tienen poco uso en oficinas de proyecto. A este respecto, también se preguntó a los ingenieros y arquitectos sobre el uso de softwares de simulación para verificar el desempeño térmico y lumínico en sus proyectos. La mayoría de los encuestados dice que no utilizan herramientas de simulación, como se puede ver en la Figura 03. Para los dos casos, 31 profesionales $(44,3 \%)$ dicen que la simulación es utilizada a veces o siempre, en cuanto que otros $39(55,7 \%)$ no han utilizado o no supieron responder.

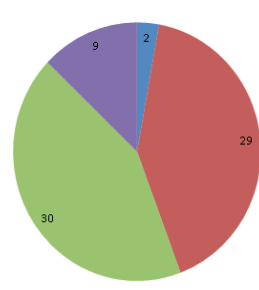

(a)

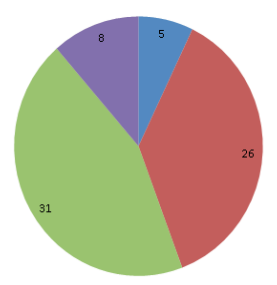

(b)
Figura 03 - Uso de software para simular el desempeño térmico (a) y uso de software de simulación para desempeño lumínico (b)

Fuente: Autores.

La falta de interés o de valoración de los contratantes es a menudo considerada como una razón para no aplicación de las estrategias de diseño que pueden ampliar el desempeño ambiental de los edificios. Los encuestados entonces respondieron a preguntas sobre el interés de sus clientes por los aspectos de desempeño térmico, acústico y lumínico. Se les preguntó con qué frecuencia los clientes solicitan estos análisis para definir estrategias de expansión de confort ambiental. Como resultado, $47(67,1 \%)$ de los encuestados respondió que sus clientes piden este tipo de análisis a veces y solo $3(4,3 \%)$ dijeron que los clientes siempre lo hacen (ver Figura 4). Por lo tanto, se puede ver que la parte del mercado ya tiene una comprensión de la importancia de aplicación de estas estrategias de diseño.

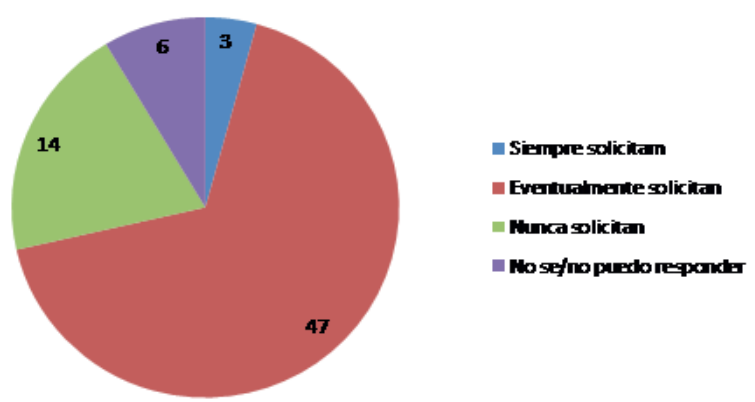

Figura 04 - Solicitud de los clientes para la implementación de estrategias para ampliar el desempeño ambiental Fuente: Autores.

Cuando se les preguntó qué elementos ya han sido ordenados por los clientes de los encuestados, las respuestas revelaron que la mejoría del desempeño térmico es el elemento más solicitado por el mercado (Figura 05).

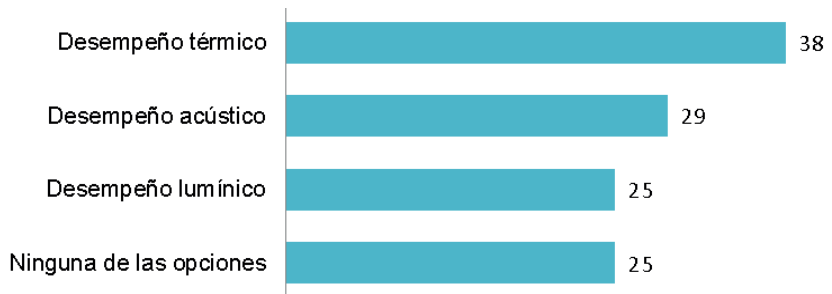

Figura 05 - Elementos relacionados con el desempeño ambiental requeridos por los clientes Fuente: Autores.

Sobre los obstáculos para la aplicación de los conceptos de la normativa NBR 15575 para la expansión del confort térmico en el interior del edificio, la mayoría de los encuestados considera que la mayor dificultad es falta de valorización de esta actividad por el mercado, seguido por el desconocimiento por parte de los propios profesionales (Figura 06).

Con la introducción de la NBR 15575, hubo necesidad de ampliar el conocimiento de los arquitectos e ingenieros civiles sobre el desempeño empieza a ser reconocido por los propios profesionales. Por lo tanto, se verifica la necesidad de un ajuste en los cursos de grado de arquitectura e ingeniería para que los nuevos profesionales tengan la oportunidad de obtener más conocimiento sobre este tema (CAUBR, 2013).

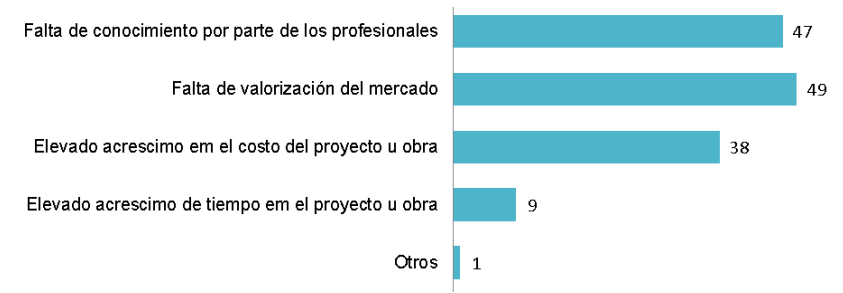

Figura 06 - Obstáculos para la implementación de estrategias de desempeño en proyectos y obras Fuente: Autores. 
Además del conocimiento de los conceptos de desempeño, es importante que el profesional sepa, por ejemplo, la forma de aplicarlos de manera eficiente en el momento del proyecto. Esto se puede facilitar con el aprendizaje de herramientas BIM, las cuales, según Barison y Santos (2016), aún están lejos de la mayoría de las universidades en Brasil.

Además, se preguntó a los encuestados sobre su conocimiento de la propia NBR 15575 (ABNT, 2013a). En ese caso, se verificó una buena situación, pues 34 profesionales $(48,6 \%)$ dicen conocer bien la norma y la misma cantidad afirma conocer una parte de la NBR 15575. Sólo 2 profesionales (un arquitecto y un ingeniero civil), afirmaron que no conocen esa normativa (Figura 07).

Por último, los encuestados respondieron sobre la importancia de hacer el análisis de impacto ambiental del edificio. De los profesionales, $87,3 \%$ consideran muy importante el análisis del desempeño térmico, seguido de un $8,9 \%$ que consideran de importancia media y $3,8 \%$ que considera de poca importancia.

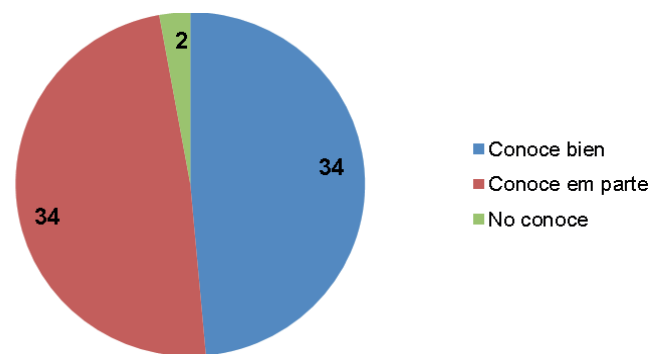

Figura 07 - Conocimiento de los profesionales sobre la NBR 15575 Fuente: Autores.

El desempeño acústico es considerado por $81 \%$ de los encuestados como un análisis muy importante; para $13,9 \%$ tiene importancia media y un $5,1 \%$ cree que sea de menor importancia.

El desempeño lumínico es visto por el $72,2 \%$ de los profesionales como de gran importancia, para 22,8\% como de importancia media y otros 5,1\% afirman ser de poca importancia. Las cifras de este análisis revelan que la mayoría de los profesionales entrevistados consideran las tres partes como muy importantes.

\section{CONCLUSIONES}

A partir de las respuestas recogidas a través de cuestionarios enviados a arquitectos e ingenieros civiles que actúan en la región de Porto Alegre, Brasil, se verificó que la mayoría de estos tienen un buen conocimiento de los conceptos de desempeño ambiental y sobre la NBR 15575 y algunos de ellos incluso ya están aplicando eses conceptos en algunos de sus proyectos.
La mayoría considera de gran importancia el análisis y la implementación de estrategias para ampliar el desempeño ambiental de los edificios, al mismo tiempo en que reconoce la necesidad de un mayor conocimiento del sector en esta área.

De acuerdo con la información recopilada también se puede verificar la preocupación por parte de algunos clientes a respecto del desempeño térmico, acústico y lumínico. Estos solicitan a menudo al diseñador que incluya el análisis y la aplicación de conceptos relacionados con estos en proyectos contratados. Aun así, la falta de valorización por el mercado fue considerada por los encuestados como un obstáculo a la inversión en edificios con un mayor desempeño.

Aunque existan retos y dificultades a superar, el desempeño ambiental es cada vez más popular entre profesionales y clientes, lo cual es de gran importancia para el desarrollo y consolidación en el mercado de la construcción.

\section{AGRADECIMIENTOS}

Los autores de este artículo agradecen a los órganos de investigación brasileños Coordenação de Aperfeiçoamento de Pessoal de Nível Superior - Brazil (CAPES) - Finance Code 001, Fundação de Amparo à Pesquisa do Estado do Rio Grande do Sul (FAPERGS) y Conselho Nacional de Pesquisa (CNPq), por el apoyo y financiación a esta investigación.

\section{REFERENCIAS}

ABNT (ASSOCIAÇÃO BRASILEIRA DE NORMAS TÉCNICAS). NBR 15215 - Iluminação Natural. Partes 1-3. Rio de Janeiro: ABNT, 2013b.

ABNT (ASSOCIAÇÃO BRASILEIRA DE NORMAS TÉCNICAS). NBR 15575 - Desempenho de edifícios habitacionais de até cinco pavimentos - Partes 1-6. Rio de Janeiro: ABNT, 2013 a.

ABNT (ASSOCIAÇÃO BRASILEIRA DE NORMAS TÉCNICAS). NBR ISO/CIE 8995 - Iluminação de ambientes de trabalho. Rio de Janeiro: ABNT, $2013 \mathrm{C}$. BARISON, M. B.; SANTOS, E. T. O papel do arquiteto em empreendimentos desenvolvidos com a tecnologia BIM e as habilidades que devem ser ensinadas na universidade. Gestão e Tecnologia de Projetos, São Carlos, v. 11, n. 1, p. 103-120, 2016. Disponible en http:// dx.doi.org/10.11606/gtp.v11i1.102708

BENTO, A. J., DAS NEVES, D. C. M., PIRES, J. M., de OLIVEIRA, M. S., DA SILVA, D. L. A influência da NBR 15575 na durabilidade e vida útil das edificações residenciais. In Seminário de Patologia e Recuperação Estrutural. Anales... Recife, Brasil: Universidade de 
Pernambuco, 2016. Disponible en http://revistas.poli. br/ anais/index.php/ semipar/article/view/228/13 CAUBR (Conselho de Arquitetura e Urbanismo do Brasil). Norma de desempenho traz grandes mudanças para construção de edifícios residenciais. Brasília: CAUBR, 2013. Disponible en http://www.caubr.gov.br/mudancasnormadesempenho/

CBIC (Câmara Brasileira da Indústria da Construção). Desempenho de Edificações Habitacionais - Guia Orientativo para Atendimento a NBR 15575/2013. Fortaleza: Gadioli Cipolla Comunicação, 2013. Disponible en http://www.cbic.org.br/arquivos/guia_ livro/Guia_CBIC_Norma_Desempenho_2_edicao.pdf CORBELLA, O.; YANNAS, S. Em busca de uma arquitetura sustentável para os trópicos: Desempenho ambiental, 2 2a ed, Revan, Rio de Janeiro, 2009.

CUNHA, E. G., ZECHMEISTER, D., MELO, E. Q., MASCARÓ, J. J., VASCONCELLOS, L de, FRANDOLOSO, M. A. L. Elementos de Arquitectura de climatização natural: método projetual buscando a Arquitectura nas edificações, 2 2a ed., Porto Alegre, Masquatro Editora, 2006. FERRAZ, R. Atenuação de ruído de impacto em pisos de edificações de múltiplos pavimentos, 2008. Dissertación (Maestria en Ingeniería) Programa de Pós Graduação em Engenharia Civil, Universidade de Minas Gerais, Belo Horizonte, 2008.

FLACH, F., PIRES, J. R., GONZÁLEZ, M. A. S., KERN, A. P. Investigação da Importância dada pelos Profissionais da Região Metropolitana de Porto Alegre, Brasil para o Desempenho Ambiental em seus Projetos. Revista AIDIS de Ingeniería y Ciencias Ambientales, v.10, p.1-17, 2017.

FROTA, A. B, SCHIFFER, S. R. Manual de desempenho térmico: 9rquitectura, urbanismo, $5^{\text {a }}$ ed., São Paulo: Studio Nobel, 2001.

GONÇALVES, O. M., JOHN, V. M., PICCHI, F. A, SATO, N. M. N. Normas Técnicas para Avaliação de Sistemas Construtivos Inovadores para Habitações. In: Roman, H., Bonin, L. C. (Eds.), Coletânea Habitare: Volume 3: Normalização e certificação na construção habitacional. Porto Alegre: ANTAC, 2007.

IPT (Instituto de Pesquisas Tecnológicas). Avaliação do desempenho térmico e energético de edificações e projetos. São Paulo: IPT, 2016. Disponible en http://www.ipt.br/solucoes/190-avaliacao_do_desempenho_termico_e_energetico_de_edificacoes_e_projetos.htm.

KERN, A. P.; SILVA, A.; KAZMIERCZAK, C. S. O processo de implantação de normas de desempenho na construção: um comparativo entre a Espanha (CTE) e Brasil (NBR 15575/2013). Gestão e Tecnologia de Projetos, v.9, n.1, p. 89-102, 2015. Disponible en http://www.revistas.usp.br/gestaodeprojetos/article/ view/89989/92778

LAMBERTS, R, GHISI, E., PEREIRA, C, D., BATISTA, J. O. Casa eficiente: Bioclimatologia e desempenho térmico. Florianópolis, Brasil: LabEEE, Universidade Federal de Santa Catarina, 2010.

LAMBERTS, R., DUTRA, L., PEREIRA, F. O. R. Eficiência Energética na Arquitetura. 3aed., Florianópolis: PW Editores, 2014.

LAMBERTS, R., GHISI, E., ABREU, A. L. P, de, CARLO, J. C., BATISTA, J, O., MARINOSKI, D. L. Desempenho térmico em edificações. Florianópolis, Brasil: LabEEE, Universidade Federal de Santa Catarina, 2007.

LOPES, R. K. Relações e influências da aplicação da acústica no processo de projeto de arquitetura contemporânea. 2010. Dissertación (Maestria en Ingeniería). Campinas: Programa de Engenharia Civil, Universidade Estadual de Campinas, 2010.

MACEDO, C. C. Análise do desempenho térmico e luminoso de sistemas de iluminação natural que utilizam a luz direta do sol. 2004. Dissertación (Maestria en Ingeniería). Florianópolis: Programa de Pós-graduação em Engenharia Civil, Universidade de Santa Catarina, 2004.

MITIDIERI FILHO, C. V.; HELENE, P.R. L. Avaliação de desempenho de componentes e elementos construtivos inovadores destinados a habitações: proposições específicas à avaliação do desempenho estrutural. São Paulo: Departamento de Engenharia de Construção Civil, Escola Politécnica da USP, 1998.

OKАMOTO, P. S. Os impactos da norma brasileira de desempenho sobre o processo de projeto de edificações residenciais. 2015, Dissertación (Maestria em Ingeniería). Departamento de Engenharia de Construção Civil, Escola Politécnica da Universidade de São Paulo, São Paulo, 2015.

OMS (Organização Mundial da Saúde). Résumé d'orientation des Directives de l'OMS Relatives au bruit dans l'environmental. Ginebra-Suiza: OMS, 2003. Disponible en http://www.who.int/ docstore/ peh/noise/bruit.htm

PALOS, S.; KIVINIEMI, A.; KUUSISTO, J. Future perspectives on product data management in building information modeling. Construction Innovation, v. 14, n. 1, p. 52-68, 2014. Disponible en http://dx.doi.org/10.1108/ Cl-12-2011-0080 


\section{AUTORES}

ORCID: https://orcid.org/0000-0001-9329-6477

BRUNA LILIANE BRENNER, Ms.c. | Universidade do Vale do Rio dos Sinos, São Leopoldo, RS - Brasil | FSG Centro Universiário da Serra Gaucha, Caxias do Sul, RS - Brasil | Correspondência para: FSG - Rua Os Dezoito do Forte, 2366 - Caxias do Sul, RS - Brasil - 95020-472 | email: brunaabrenner@gmail.com

ORCID: https://orcid.org/0000-0002-4115-3578

MATHEUS SBARDELOTTO BALDO | Universidade do Vale do Rio dos Sinos, São Leopoldo, RS - Brasil | Correspondência para: UNISINOS - Av. Unisinos, 950 - São Leopoldo, RS Brasil - 93022-001| email: matheusbaldo@gmail.com

ORCID: http://orcid.org/0000-0002-1975-0026

MARCO AURELIO StUMPF GONZaLeZ, Dr. | Professor - PPGEC - Escola Politécnica - Universidade do Vale do Rio dos Sinos, São Leopoldo, RS - Brasil | Correspondência para: UNISINOS - Av. Unisinos, 950 - São Leopoldo, RS - Brasil 93022-001 | email:mgonzalez@unisinos.br

ORCID: http://orcid.org/0000-0001-6406-6250

ANDREA PARISI KERN, Dra. | Professora - PPGEC - Escola Politécnica - Universidade do Vale do Rio dos Sinos, São Leopoldo, RS - Brasil | Correspondência para: UNISINOS - Av. Unisinos, 950 - São Leopoldo, RS - Brasil - 93022-001| email: apkern@unisinos.br

\section{COMO CITAR ESTE ARTIGO}

BRENNER, Bruna Liliane; BALDO, Matheus Sbardelotto; GONZALEZ, Marco Aurelio Stumpf; KERN, Andrea Parisi. Análisis de la Aplicación da la Normativa Brasileña NBR 15575 y Algunas Estrategias para Aumentar la Calidad Ambiental en Edificios Habitacionales. MIX Sustentável, [S.I.], v. 5, n. 4, p. 71-78, out. 2019. ISSN 24473073. Disponível em:<http://www.nexos.ufsc. br/index.php/mixsustentavel>. Acesso em: dia mês. ano. doi:https://doi.org/10.29183/2447-3073.MIX2019. v5.n4.71-78 